\title{
Identification of Microorganisms Using Superconducting Tunnel Junctions and Time-of-Flight Mass Spectrometry
}

\author{
J.N. Ullom, M. Frank, J.M. Horn, S.E. Labov, K. Langry, \\ W.H. Benner
}

This article was submitted to

Eighth International Workshop on Low Temperature Detectors LTD8, Dalfsen, The Netherlands, August 15-20, 1999

\section{August 30, 1999}

U.S. Department of Energy

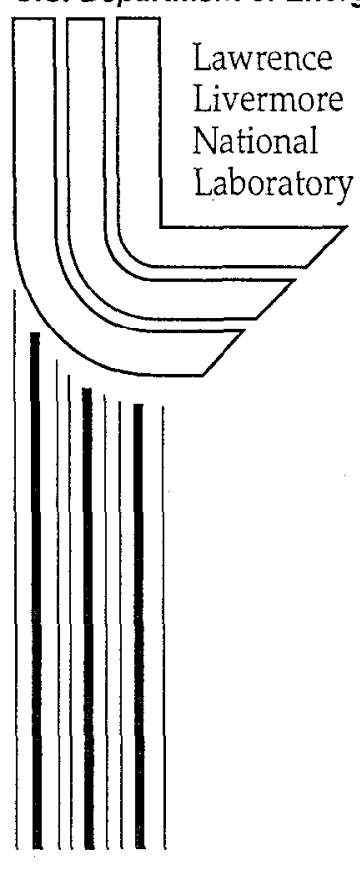




\section{DISCLAIMER}

This document was prepared as an account of work sponsored by an agency of the United States Government. Neither the United States Government nor the University of California nor any of their employees, makes any warranty, express or implied, or assumes any legal liability or responsibility for the accuracy, completeness, or usefulness of any information, apparatus, product, or process

disclosed, or represents that its use would not infringe privately owned rights. Reference herein to any specific commercial product, process, or service by trade name, trademark, manufacturer, or otherwise, does not necessarily constitute or imply its endorsement, recommendation, or favoring by the United States Government or the University of California. The views and opinions of authors expressed herein do not necessarily state or reflect those of the United States Government or the University of California, and shall not be used for advertising or product endorsement purposes. 


\title{
IDENTIFICATION OF MICROORGANISMS USING SUPERCONDUCTING TUNNEL JUNCTIONS AND TIME-OF-FLIGHT MASS SPECTROMETRY
}

\author{
J.N. Ullum, M. Frank ${ }^{a}$, J.M. Horn', S.E. Labov ${ }^{2}$, K. Langry ${ }^{a}$, W.H. Benner ${ }^{b}$ \\ ${ }^{2}$ Lawrence Livermore National Laboratory, Livermore CA, 94550, USA \\ "Lawrence Berkeley National Laboratory, Berkeley CA, 94720, USA
}

\begin{abstract}
We present time-of-flight measurements of hiological material ejected from complete bacterial spores following laser irradiation. Ion impacts are registered on a microchannel plate and on a Superconducting Tunnel Junction (STJ) detector. We compare mass spectra obtained with the two detectors. The STJ has better sensitivity to massive ions and also measures the energy of each ion. We show evidence that spores of different bacillus species produce distinctive mass spectra and associate the observed mass peaks with coat proteins identified through ion-exchange extraction and gel electrophoresis.
\end{abstract}

\section{INTRODUCTION}

A rapid and robust technique for identifying microorganisms is desirable for a wide range of environmental and medical applications. The suitability of mass spectrometry has already been demonstrated. [1] In this paper, we describe ongoing efforts to identify microorganisms using timeof-flight mass spectrometry and superconducting tunnel junction detectors. The use of cryogenic detectors for mass spectrometry is relatively new [2-4] but they have a number of properties which merit exploration. First, the sensitivity of cryogenic detectors is expected to be independent of ion mass, unlike detectors based on secondary electron emission whose sensitivity decreases for heavier, slower ions. In addition, cryogenic detectors provide pulse-heightenergy information for each incident ion. This information allows discrimination between charge states and can also be used to probe launch conditions.

\section{TECHNIQUE}

We have concentrated on understanding the mass spectra produced by whole bacterial spores. A typical spore has a diameter of approximately $1 \mu \mathrm{m}$ and weighs about $10^{12}$ Daltons $\left(10^{12} \mathrm{~m}_{\mathrm{p}}\right)$. A target is prepared by mixing many spores with a light, UV absorbing molecule called the matrix. We chose sinapinic acid (224 Da) for the matrix. The resulting solution is air-dried and inserted into a linear time-offlight mass spectrometer.

Ions are generated and launched from the target by a pulse from a UV laser. Most ions are singly charged but some higher charge states are present. We used a $337 \mathrm{~nm}$ nitrogen laser which produced 3 ns pulses of about $100 \mu \mathrm{J}$ each.

Ions are accelerated through a $30 \mathrm{kV}$ potential difference over a distance of $3 \mathrm{~cm}$ and then undergo free flight for $1.7 \mathrm{~m}$. The ions then enter a small, pumped helium cryostat and impact on the top electrode of a superconducting tunnel junction (STJ) detector. The junction is a $\mathrm{Nb}-\mathrm{AlOx}-\mathrm{Nb}$ trilayer with an area of $0.04 \mathrm{~mm}^{2}$. We have used detectors both with and without a SiOx passivation layer. In either case, the impact of an ion produces phonons which create quasiparticles in the top electrode of the 
junction. The tunneling of quasiparticles between the junction electrodes produces a measurable current pulse. The amplitude of this pulse is related to the energy of the incident ion and the time between the laser pulse and the onset of the detector response is the ion flight time. In principle, the mass and charge of each ion can be uniquely determined from measurements of its energy and flight time.

\section{RESULTS}

Data obtained from whole spores of Bacillus Cereus is shown in Fig. 1. Pulse heights from singly charged ions fall below about $15 \mathrm{mV}$. The cluster of ions arriving before $50 \mu$ s corresponds to monomers, dimers, etc. of the matrix. It can readily be seen that, despite having the same kinetic energy, singly charged ions produce a range of pulse heights.

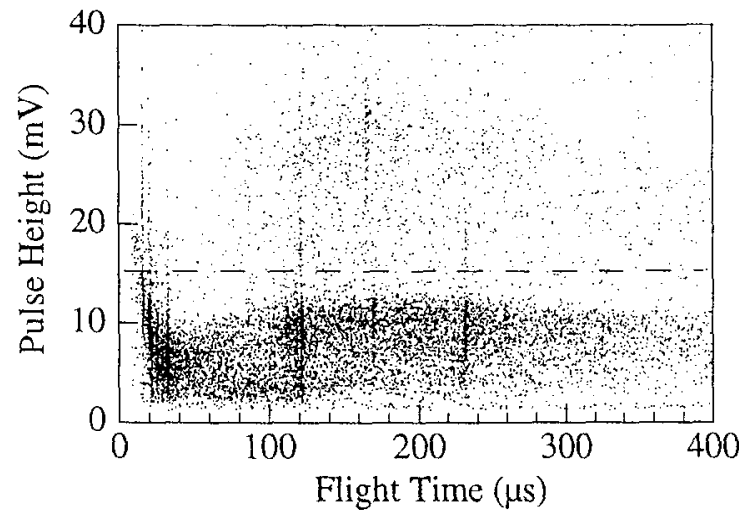

fig. 1 Pulse heights and flight times from B. Cereus. Each point is a single ion striking the detector. This plot represents about 5000 laser pulses.

Early arriving (light) ions produce the largest pulses and there is a minimum in the detector response for ions arriving at about $40 \mu \mathrm{s}$. This structure is not well understood but we believe the deposited energy depends on a number of factors including fragmentation of the incident ion, internal energy carried by the incident ion, and damage done to the detector surface. Clusters of ions arriving at 120, 170,230 , and $260 \mu$ s are characteristic fragments from the bacterial spores. The longest of these flight times corresponds to an ion mass of $91 \mathrm{kDa}$ so each fragment represents only a small part of a spore.

Ion exchange extraction was used to isolate coat proteins of $B$. Cereus and these proteins were used as a separate target in the mass spectrometer. The measured flight times were identical to those observed from whole spores except the $260 \mu$ s ion peak was absent from the coat protein spectrum. We conclude that the fragments launched from the whole spores are coat proteins. Gel electrophoresis was also performed on the extracted coat proteins. The ion Inasses deduced from the electropherogram were similar but not identical to those deduced from the flight times. It is not surprising that quantitative differences exist since electrophoresis measures ion drag which is not a perfect analog to mass. We conclude that the ion masses measured using the STJ detector and time-of-flight mass spectrometry are accurate. It should be noted that the peak in the flight spectrum at $170 \mu \mathrm{s}$ was not present in the electropherogram.

We also took data from B. Cereus using a conventional microchannel plate detector (MCP). Histograms of ion mass acquired with the STJ and MCP are shown in Fig. 2. Ion mass is calculated assuming a charge of unity. The sensitivity of the MCP is reduced for heavier, slower ions. As a result, the MCP struggles to detect the $72.6 \mathrm{kDa}$ ions and fails to detect the $91.0 \mathrm{kDa}$ ions. In contrast, both are readily apparent in the STJ spectrum. In addition, the ability of the STJ to measure ion charge allows the peak at $38 \mathrm{kDa}$ to be separated into doubly charged ions of $72.6 \mathrm{kDa}$ and singly charged ions of 38.2 $\mathrm{kDa}$. The MCP provides no charge information so the user cannot know with certainty that a singly charged species is present in the $38 \mathrm{kDa}$ peak. While this is not an exhaustive comparison, we believe this example highlights the advantages of cryogenic detectors for mass spectrometry.

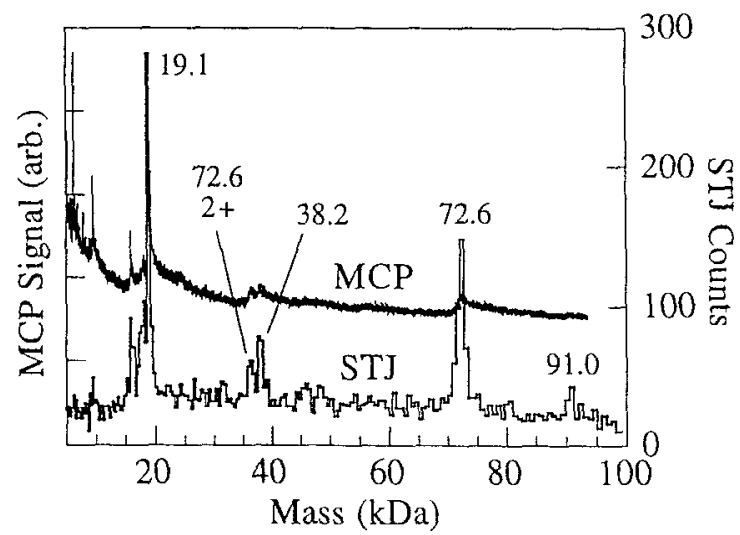

fig. 2 Mass spectra of B. Cereus obtained with STJ and $M C P$ detectors.

In Fig. 3, histograms of ion flight time obtained with the STJ are plotted for targets of $B$. Cereus and B. Subtilis. The two spectra are clearly different demonstrating that bacterial species can be distinguished hy mass spectrometry. 


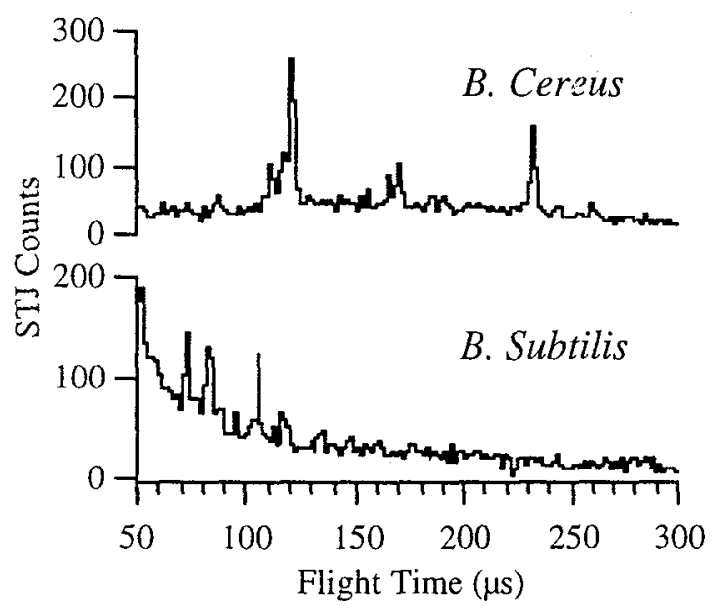

fig. 3 Ion flight times for B. Cereus and B. Subtilis. Flight time is displayed instead of mass in order to emphasize peaks at short times.

The highest useful sensitivity level is the detection of a single spore with a single laser pulse. From estimates of the density of spores in the target, we believe we liberate $10^{-4}-10^{-3}$ ions/pulse/spore. Since multiple ions are required to form a spectrum, it is clear that we presently fall many orders of magnitude short of this sensitivity level. In the future, we plan to explore infrared laser wavelengths in order to improve the ion yield per pulse. Our sensitivity can be further improved by using an array of STJs to capture more ions from each laser pulse. (Note that a single large detector is less useful because the ions of interest arrive at similar times and this pileup degrades the energy resolution.) If the ion masses are below about $40 \mathrm{kDa}$, and if pulse height information is not needed, use of a large area microchannel plate will improve the ion yield by about 3 orders of magnitude. However, for ion masses approaching $100 \mathrm{kDa}$, the $\mathrm{MCP}$ sensitivity falls by 2 orders of magnitude, negating much of this gain. [5]

\section{CONCLUSIONS}

We have used laser irradiation to ablate and launch material from whole bacterial spores. We have detected the spore fragments by impacting them on a superconducting tunnel junction detector. We have confirmed that the fragments are spore coat proteins and that the measured fragment masses are accurate. We have compared mass spectra obtained with the tunnel junction and with a microchannel plate. The tunnel junction yields additional information about high mass fragments and its sensitivity to ion charge clarifies interpretation of the mass spectrum. Finally, we have successfully differentiated two bacterial species based on their mass spectra.

This work was performed under the auspices of the U.S. Department of Fnergy by Lawrence Livermore National Laboratory under contract No. W-7405-ENG-48 and supported by the Director, Office of Energy Research, Office of Health and Environmental Research, Human Genome Project, U.S. Department of Energy under contract no. DEAC03-76SF00098.

\section{REFERENCES}

[1] See, for example, J.P. Anhalt, C. Fenselau, Anal. Chem. 47 (1975) 219 or A. Haag, S. Taylor, K. Johnston, R. Cole, J. Mass Spectrom. 33 (1998) 750.

[2] D. Twerenbold, Nucl. Instr. and Meth. A. 370 (1996) 253.

[3] G.C. Hilton, J.M. Martinis, D.A. Wollman, K.D. Irwin, et al, Nature 391 (1998) 672.

[4] M. Frank, S.E. Labov, G. Westmacott, W.H. Benner, Mass. Spectr. Rev. (in press)

[5] G. Westmacott, W.H. Benner, M. Frank, S.E. Labov (in preparation) 